\title{
NATURDATA.DK - Danish monitoring program of vegetation and chemical plant and soil data from non-forested terrestrial habitat types
}

\author{
Knud Erik Nielsen, J.L. Bak, M. Bruus, C. Damgaard, R. Ejrnæs, J.R. Fredshavn, B. \\ Nygaard, F. Skov, B. Strandberg \& M. Strandberg
}

\begin{abstract}
Vegetation and chemical plant and soil data from 18 terrestrial non-forested natural habitat types have been collected each year since 2004 (Table 1). Presently, cover data of plant species measured by pinpoint (16 grid points in $0.5 \mathrm{~m} \mathrm{x} 0.5 \mathrm{~m}$ frame) and plant frequency data $(5 \mathrm{~m}$ circles) exist for more than 50,000 plots; the measuring of chemical properties in plant, soil, and water samples has been less intense. Presently there are more than 12,000 registrations of carbon content and 57,000 measurements of $\mathrm{pH}$ in topsoil. Soils are always samples from the top $5 \mathrm{~cm}$. On heathland habitats the thickness of the mor layer is measured. All data are georeferenced and can be accessed at http://www.naturdata.dk/ The data comprises approximately 1,200 sites both within and outside of Natura 2000 areas. At each site the measurements were made at 20-60 randomly positioned plots. $70 \%$ of the plots have been revisited each year, whereas $30 \%$ have only been visited once. Each year a total number of additional 5,000 sample plots and registrations will be performed. The protocols for the different measurement types (in Danish) may be downloaded from http://www.dmu.dk/fileadmin/Attachments/TAN1_106_01_FDCNY1.pdf. This report describes the available content in the vegetation-plot database NATURDATA.DK (GIVD ID EU-DK-001).
\end{abstract}

Keywords: moss; nitrogen; pin point; plant cover; plant frequency; soil analysis.

\section{NATURDATA.DK}

Scope: Cover and frequency of vegetation and chemical plant and soil data from 18 terrestrial non-forested natural habitat types in Denmark. Status: ongoing capture Period: 2004-2010

Database manager(s): Knud Erik Nielsen (ken@dmu.dk); J.L. Bak (jlb@dmu.dk); M. Bruus (mbp@dmu.dk)

Owner: The Danish Nature \& Environment Portal

Web address: http://www.miljoeportal.dk/English

Availability: free upon request

Online upload: yes

Online search: yes

Database format(s): MySQL

Export format(s): SQL

Publication: [NA]

Plot type(s): nested plots

Plot-size range: $0,5-78 \mathrm{~m}^{2}$

Non-overlapping plots: 1,200

Total plot observations: 50,000

Estimate of existing plots: [NA]

Number of sources: 1

\section{Completeness: [NA]}

Valid taxa: [NA]

Countries: DK: $100.0 \%$

Forest: [NA] — Non-forest: [NA]

Guilds: all vascular plants: $100 \%$

Environmental data: altitude: $100 \%$; slope aspect: $100 \%$; slope inclination: $100 \%$; soil depth: $100 \%$; surface cover other than plants (open soil, litter, bare rock etc.): $25 \%$; soil $\mathrm{pH}: 25 \%$; land use categories: $10 \%$

Performance measure(s): presence/absence only: $50 \%$; cover: $50 \%$

Geographic localisation: GPS coordinates (precision $25 \mathrm{~m}$ or less): $100 \%$

Sampling periods: [NA]

Information as of 2012-07-12; further details and future updates available from http://www.givd.info/ID/EU-DK-001

Knud Erik Nielsen* (ken@dmu.dk), J.L. Bak (jlb@dmu.dk), M. Bruus (mbp@dmu.dk), C. Damgaard (cfd@dmu.dk), R. Ejrnæs (rej@dmu.dk), J.R. Fredshavn (jrf@dmu.dk), B. Nygaard (bny@dmu.dk), F. Skov (fs@dmu.dk), B. Strandberg (bst@dmu.dk), M. Strandberg (mts@dmu.dk)

Institute of Bioscience, Aarhus University, Vejlsøvej 25, 8600 Silkeborg, DENMARK

*Corresponding author 